\title{
Kinetics of binding and diffusivity of leucine-enkephalin in large unilamellar vesicle by pulsed-field-gradient ${ }^{1} \mathrm{H}$ NMR in situ
}

\author{
Noriyuki Yoshii $^{1,2}$, Tomomi Emoto ${ }^{1}$ and Emiko Okamura ${ }^{1}$ \\ ${ }^{1}$ Faculty of Pharmaceutical Sciences, Himeji Dokkyo University, 7-2-1, Kamiohno, Himeji 670-8524, Japan \\ ${ }^{2}$ Present address: Graduate School of Engineering, Nagoya University
}

Received August 30, 2011; accepted October 18, 2011

\begin{abstract}
The kinetics of binding, the diffusivity, and the binding amount of a neuropeptide, leucine-enkephalin (L-Enk) to lipid bilayer membranes are quantified by pulsedfield-gradient (PFG) ${ }^{1} \mathrm{H}$ NMR in situ. The peptide signal is analyzed by the solution of the Bloch equation with exchange terms in the presence of large unilamellar vesicles (LUVs) as confined, but fluid model cell membranes. Even in the case that the membrane-bound and the free states of L-Enk cannot be distinguished in the one-dimensional NMR spectrum, the PFG technique unveils the bound component of $L$-Enk after the preferential decay of the free component at the high field gradient. In 100-nm diameter LUVs consisting of egg phosphatidylcholine, the rate constants of the peptide binding and dissociation are 0.040 and $0.40 \mathrm{~s}^{-1}$ at $303 \mathrm{~K}$. This means that the lifetime of the peptide binding is of the order from second to ten-second. The diffusivity of the bound L-Enk is $5 \times 10^{-12} \mathrm{~m}^{2} / \mathrm{s}$, almost 60 times as restricted as the movement of free L-Enk at $303 \mathrm{~K}$. Onetenth of $5 \mathrm{mM}$ L-Enk is bound to $40 \mathrm{mM}$ LUV. The binding free energy is calculated to be $-2.9 \mathrm{~kJ} / \mathrm{mol}$, the magnitude close to the thermal fluctuation, $2.5 \mathrm{~kJ} / \mathrm{mol}$. The result demonstrates the potential of PFG ${ }^{1} \mathrm{H}$ NMR to quantify molecular dynamics of the peptide binding to membranes.
\end{abstract}

Key words: exchange, mobility,

fluid membrane dynamics, drug delivery, free energy of transfer from water to membrane

Corresponding author: Emiko Okamura, Faculty of Pharmaceutical Sciences, Himeji Dokkyo University, 7-2-1, Kamiohno, Himeji 6708524, Japan. e-mail: emiko@himeji-du.ac.jp

\section{Introduction}

Molecular dynamics of peptide binding to fluid lipid membranes is crucial for a better understanding of the primary stage of the bioactivities in the cell. In situ technique is powerful to gain insight into the mechanistic view of peptide functions in a natural manner. Pulsed-field-gradient (PFG) NMR spectroscopy ${ }^{1}$ is a versatile method to measure the molecular diffusion without disturbing the system. Recently we have applied high-resolution solution-state ${ }^{19} \mathrm{~F}$ NMR in combination with the PFG technique, to study the drug binding and mobility in confined but fluid membranes ${ }^{2-4}$. We have developed the strategy to quantify how much and how fast the drug is bound to the membrane and how fast the drug is moving in the membrane without the use of labeled nuclei or the aid of sample spinning to obtain wellresolved spectra of high $\mathrm{S} / \mathrm{N}$ ratio. The previous studies have adopted the small-sized 5-fluorouracil (5FU, 5-fluoro$1 \mathrm{H}$-pyrimidine-2,4-dione) and fluorinated bisphenol $\mathrm{A}$ (FBPA, $\left.\left(\mathrm{CF}_{3}\right)_{2} \mathrm{C}\left(\mathrm{C}_{6} \mathrm{H}_{4} \mathrm{OH}\right)_{2}\right)$ as a drug model. Fluorine-19 NMR is advantageous to observe the drug side because the ${ }^{19} \mathrm{~F}$ nucleus is absent in natural and model cell membranes ${ }^{5,6}$. The ${ }^{19} \mathrm{~F}$ nucleus is, however, rare in the peptide. The general method is required to quantify a wide variety of peptide bindings. Proton NMR is effective for this purpose because ${ }^{1} \mathrm{H}$ is abundant in the natural system. In this work, the PFG ${ }^{1} \mathrm{H}$ NMR method is applied to explore the dynamic aspects of the peptide binding. The diffusivity, the binding amount, and the rate constants of the binding and dissociation of a neuropeptide, leucine-enkephalin (L-Enk, Tyr-Gly-Gly-PheLeu) are quantified in phospholipid large unilamellar vesicles (LUVs) as confined but soft membrane environments. 
In the previous work, we have elucidated the exchange motion of 5FU molecules between the bound and the free states by using $\mathrm{PFG}^{19} \mathrm{~F} \mathrm{NMR}^{3}$. The analysis includes (1) the decomposition of 5FU signal into the bound and free components, (2) the evaluation of the diffusion coefficients by the Stejskal-Tanner plot $^{7}$, and (3) the determination of the exchange rate constants by the analytical formula of the Bloch equation with exchange terms ${ }^{8}$. In this case, the bound and the free components are not so largely overlapping and can be resolved in the one-dimensional (1D) spectrum to analyze the signal intensity by peak height. Such analysis is, however, impossible when the two components are hard to resolve, as shown in the ${ }^{1} \mathrm{H}$ NMR spectrum in the presence of the membrane ${ }^{2}$. Here we show that the PFG technique enables to quantify the dynamics of the peptide binding, even in the case that the bound and the free states cannot be distinguished in the 1D NMR spectrum.

Molecular study of enkephalins in model membranes has attracted great interest in relation to the analgesic effect of endogeneous neuropeptides, because the interaction of the peptide with membranes is thought to play a significant role in the subsequent receptor binding ${ }^{9-11}$. The NMR studies have focused on the conformation of enkephalins in membranemimicking environments involving micelles, bicelles, and vesicles $^{9,11-18}$. The dynamic aspects are, however, limited to the micellar system ${ }^{18-20}$, the dynamical structure different from the bilayer membrane ${ }^{21}$. Here we elucidate how fast L-Enk binds to the membrane, dissociates from the membrane, and moves in the membrane, using bilayer vesicle as a model cell membrane.

\section{Experimental}

\subsection{Sample}

In this work, LUVs rather than micelles were employed as model cell membranes in view of the dynamical structure ${ }^{21}$. As the phospholipid component, egg phosphatidylcholine (EPC, MW $\sim 770$ and $98.5 \%$ pure) was purchased from NOF CORPORATION (Tokyo, Japan) and used without further purification. L-Enk (purity, 99\%; MW 555.62) was obtained from Peptide Institute Inc. (Osaka, Japan). The heavy water solvent $\left(\mathrm{D}_{2} \mathrm{O}, 99.9 \% \mathrm{D}\right)$ was obtained from Euriso-top (Saint Aubin, France).

LUVs were prepared by an extrusion technique ${ }^{22}$ by using Mini-Extruder (Avanti Polar Lipids, Inc., AL) with a polycarbonate membrane. The size was systematically controlled by using 100-nm pore size filter. The concentration of EPC LUV was $40 \mathrm{mM}$. $\mathrm{D}_{2} \mathrm{O}$ was used as a solvent to suppress the ${ }^{1} \mathrm{H}$ NMR signal of light water. The injection of L-Enk into the LUV was performed by adding the desired amount of aqueous L-Enk solution to the vesicle suspension. The final concentration of L-Enk was fixed to $5 \mathrm{mM}$.

\subsection{NMR Measurement}

${ }^{1} \mathrm{H}$ NMR measurements were carried out at $399.8 \mathrm{MHz}$, by using a JEOL ECA400 NMR spectrometer equipped with a superconducting magnet of $9.4 \mathrm{~T}$. Although $\mathrm{D}_{2} \mathrm{O}$ was selected as a solvent, the DANTE presaturation pulse sequence was applied to avoid the signal overlapping of impurity light water (HDO) with the target peak. The presaturation was minimal so that the peptide resonance was not perturbed. The digital resolution was $0.5 \mathrm{~Hz}(0.001 \mathrm{ppm})$. Chemical shifts of the ${ }^{1} \mathrm{H}$ NMR signals were obtained by referring to the absorption frequency of the solvent deuteron monitored as the lock signal. The PFG NMR measurement was performed by using a high-sensitivity multinuclear probe (JEOL, NM-40TH5AT/FG2) under a current of $30 \mathrm{~A}$. The probe can exert a field gradient (FG) up to $0.91 \mathrm{~T} / \mathrm{m}$, most suitable for the simultaneous observation of bound and free components of the peptide. To attenuate the spin-echo signal, the 16 different FG strengths $g$ were applied in the range from 0.05 to $0.91 \mathrm{~T} / \mathrm{m}$. The linearity of the pulsed field gradient was confirmed by the diffusion measurement of benzene in $\mathrm{D}_{2} \mathrm{O}^{23}$. Bipolar Pulse Pairs (BPP)-STimulated Echo (STE)Longitudinal Eddy current Delay (LED) pulse sequence ${ }^{2}$ was adopted to minimize the influence of eddy current. The height of the sample solution was less than $5 \mathrm{~mm}$ to avoid the convection effect. In order to evaluate the accuracy of the results, the PFG measurements have been done at three different diffusion times $\left(\Delta_{\text {diff }}=0.1,0.5\right.$, and $\left.1.0 \mathrm{~s}\right)$. The pulse width $\delta$ was ranging from 0.00125 to $0.004 \mathrm{~s}$, except for the measurement of the peptide in water, 0.0003 to $0.002 \mathrm{~s}$. The 1024 FID signals were accumulated with a recycling delay of $10 \mathrm{~s}$, sufficiently longer than the longitudinal relaxation time of the peptide. For peptide solution, 32 transients were accumulated with a recycling delay of $15 \mathrm{~s}$. The measurements were carried out at $303 \mathrm{~K}$ where lipids were in the liquid-crystalline phase. The binding of L-Enk was under equilibrium, according to the NMR observation that no variation of the bound and the free signals of L-Enk was found throughout the measurement.

To ensure that the NMR signal intensity of the peptide is not influenced by the differences in the relaxation between bound (B) and free (F) components, we have calculated and made a comparison of the damping factors of relaxation ${ }^{1}$ between these two components of the peptide signal. In BPP-STE-LED pulse sequence (Fig. 3 in Ref. 2), the period $t_{\mathrm{L}}$ to compete with the longitudinal relaxation is during the time interval $T+T_{\mathrm{e}}$. The $t_{\mathrm{L}}$ was $0.15-1.05 \mathrm{~s}$ under the experimental conditions $\Delta_{\text {diff }}$ of $0.1-1.0 \mathrm{~s}$. Since the longitudinal relaxation times $T_{1}$ observed were $T_{1 \mathrm{~B}}=1.2$ and $T_{1 \mathrm{~F}}=2.3 \mathrm{~s}$ for $\mathrm{B}$ and $\mathrm{F}$ components ${ }^{24}$, the ratio of the damping factors $e^{-t_{\mathrm{L}} / T_{\mathrm{IB}}} / e^{-t_{\mathrm{L}} / T_{\mathrm{IF}}}$ was $0.63-0.94$. On the other hand, the period $t_{\mathrm{T}}$ to compete with the transverse relaxation, i.e., the twice of the interval of $90^{\circ}-180^{\circ}-90^{\circ} \mathrm{RF}$ pulse sequence, was $0.003-0.009 \mathrm{~s}$. It was short enough as compared with the transverse relaxation times, $T_{2 \mathrm{~B}}=0.078$ and $T_{2 \mathrm{~F}}=0.095 \mathrm{~s}^{24}$, to yield the ratio $e^{-t_{\mathrm{T}} / T_{2 \mathrm{~B}}} / e^{-t_{\mathrm{T}} / T_{2 \mathrm{~F}}}$ of $0.98-0.99$. Thus the influence of the relaxation was found to be within the experimental error. 


\section{Data Analysis}

\subsection{Exchange Rate Constant}

In equilibrium, the relation

$$
k_{\mathrm{BF}} N_{\mathrm{B}}=k_{\mathrm{FB}} N_{\mathrm{F}}
$$

is held, where $k_{\mathrm{FB}}$ and $k_{\mathrm{BF}}$ denote the rate constants of the peptide binding and dissociation, and $N_{\mathrm{B}}$ and $N_{\mathrm{F}}$ represent the number of bound and free peptide molecules.

\subsection{Analysis of the Peak Integral}

As shown in the ${ }^{1} \mathrm{H}$ NMR spectrum (Fig. 1), it is difficult to distinguish the bound and free components of L-Enk; see the detail in section 4.1. In this case, the individual peak height analysis is impossible, and hence the peak integral

$$
A(g)=\int_{\omega_{1}}^{\omega_{2}} I(g, \omega) d \omega
$$

that is in proportion to the peak height of the signal, is substituted for the signal intensity. In Eq. (2), $g$ and $\omega$ are the FG strength and the chemical shift, respectively. $\omega_{1}$ and $\omega_{2}$ are the lower and upper limit of the integral. Both bound (B) and free (F) components contribute to the integral intensity.

The integral intensity $A(g)$ of the PFG signal is analyzed by the analytical formula of the Bloch equation with exchange terms ${ }^{8}$. The intensity $A(g)$ can be written as

$$
\begin{aligned}
A(g)= & \left(\frac{A_{0}}{2}+\frac{\Lambda}{2 \Delta}\right) \exp [(-\sigma+\Delta) \tau] \\
& +\left(\frac{A_{0}}{2}-\frac{\Lambda}{2 \Delta}\right) \exp [(-\sigma-\Delta) \tau] .
\end{aligned}
$$

The parameters shown in Eq. (3) are defined as

$$
\Lambda=\lambda\left(A_{\mathrm{B} 0}-A_{\mathrm{F} 0}\right)+k_{\mathrm{FB}} A_{\mathrm{F} 0}+k_{\mathrm{BF}} A_{\mathrm{B} 0},
$$

where $\lambda=\frac{1}{2}\left(k_{\mathrm{FB}}-k_{\mathrm{BF}}+D_{\mathrm{F}} m^{2}-D_{\mathrm{B}} m^{2}\right)$,

$$
\begin{aligned}
& \sigma=\frac{1}{2}\left(k_{\mathrm{FB}}+k_{\mathrm{BF}}+D_{\mathrm{F}} m^{2}+D_{\mathrm{B}} m^{2}\right), \\
& \Delta=\sqrt{\lambda^{2}+k_{\mathrm{FB}} k_{\mathrm{BF}}}
\end{aligned}
$$

and

$$
\tau=\left(\Delta_{\text {diff }}-\frac{\delta}{3}\right) .
$$

$A_{\mathrm{F} 0}$ and $A_{\mathrm{B} 0}$ are defined as the peak integrals $A_{\mathrm{F}}(g)$ and $A_{\mathrm{B}}(g)$ at $\tau=0$, and

$$
A_{0}=A_{\mathrm{F} 0}+A_{\mathrm{B} 0} .
$$

$D_{\mathrm{F}}$ and $D_{\mathrm{B}}$ are the diffusion coefficients of free and bound peptides, respectively. In this work, the FG strength $g$ is reduced as

$$
m=\delta \gamma g
$$

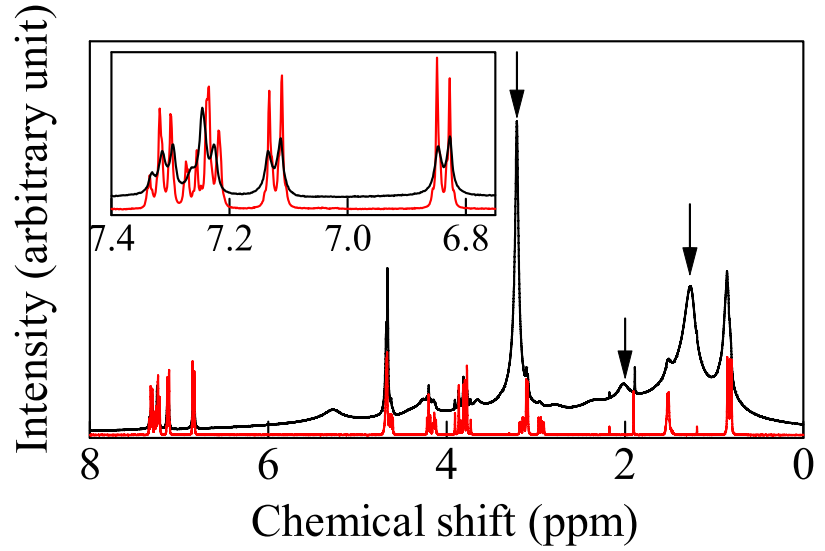

Figure 1 1D ${ }^{1} \mathrm{H}$ NMR spectra of $5 \mathrm{mM}$ L-Enk in the presence (black) and absence (red) of EPC LUV. The aromatic region of the Tyr and Phe residues of L-Enk is expanded in the inset. Arrows show the EPC signals used for determining the diffusion coefficient of the lipid in membrane.

where $\gamma$ is the gyromagnetic ratio of the ${ }^{1} \mathrm{H}$ nucleus $\left(2.675 \times 10^{8} \mathrm{rad} / \mathrm{Ts}\right)$.

Once Eq. (3) is successfully fitted to the experimental $A(g)$, the intensities of the bound and free components $A_{\mathrm{B}}(g)$ and $A_{\mathrm{F}}(g)$ can be easily evaluated by

$$
\begin{aligned}
A_{\mathrm{B}}(g)= & \left\{\frac{A_{\mathrm{B} 0}}{2}+\frac{\lambda A_{\mathrm{B} 0}+k_{\mathrm{FB}} A_{\mathrm{F} 0}}{2 \Delta}\right\} \exp \{(-\sigma+\Delta) \tau\} \\
& +\left\{\frac{A_{\mathrm{B} 0}}{2}-\frac{\lambda A_{\mathrm{B} 0}+k_{\mathrm{FB}} A_{\mathrm{F} 0}}{2 \Delta}\right\} \exp \{(-\sigma-\Delta) \tau\},
\end{aligned}
$$

and

$$
\begin{aligned}
A_{\mathrm{F}}(g)= & \left\{\frac{A_{\mathrm{F} 0}}{2}-\frac{\lambda A_{\mathrm{F} 0}-k_{\mathrm{BF}} A_{\mathrm{B} 0}}{2 \Delta}\right\} \exp \{(-\sigma+\Delta) \tau\} \\
& +\left\{\frac{A_{\mathrm{F} 0}}{2}+\frac{\lambda A_{\mathrm{F} 0}-k_{\mathrm{BF}} A_{\mathrm{B} 0}}{2 \Delta}\right\} \exp \{(-\sigma-\Delta) \tau\} .
\end{aligned}
$$

\subsection{Fitting Procedure}

There are 6 parameters, $k_{\mathrm{BF}}, k_{\mathrm{FB}}, D_{\mathrm{B}}, D_{\mathrm{F}}, A_{\mathrm{B} 0}$, and $A_{\mathrm{F} 0}$ in Eq. (3). $A_{\mathrm{B} 0}$ and $A_{\mathrm{F} 0}$ are in proportion to the number of bound and free molecules, respectively. From Eq. (1), we can write the similar relation as

$$
k_{\mathrm{FB}} A_{\mathrm{F} 0}=k_{\mathrm{BF}} A_{\mathrm{B} 0} .
$$

When we obtain the experimental $A_{0}$, the two parameters $A_{\mathrm{B} 0}$ and $A_{\mathrm{F} 0}$ can be removed from Eq. (3) by using Eqs. (8) and (12).

At large $g$, the Stejskal-Tanner plot clearly shows that the intensity is a linear function of $g^{2}$, so that it is possible to obtain $D_{\mathrm{B}}$ by fitting the equation 


$$
\ln \frac{A(g)}{A_{0}}=-D_{\mathrm{B}} m^{2} \tau
$$

to the experimental $A(g)$; the detail will be described in section 4.2. Thus, the fitting parameters can be reduced to $k_{\mathrm{FB}}$, $k_{\mathrm{BF}}$, and $D_{\mathrm{F}}$. Finally, we have only to fit Eq. (3) to the experimental $A(g)$ in the whole range of $g$ and evaluate $k_{\mathrm{FB}}, k_{\mathrm{BF}}$, and $D_{\mathrm{F}}$ simultaneously.

\section{Results and Discussion}

\subsection{D and PFG ${ }^{1} \mathrm{H}$ NMR Spectra of L-Enk in the Presence of Vesicle}

First we examine how the NMR spectrum of L-Enk is modified by the presence of the vesicle. Figure 1 shows the 1D ${ }^{1} \mathrm{H}$ NMR spectra of L-Enk in the presence and absence of EPC LUV with 100-nm diameters. The aromatic proton region at 7.4-6.7 ppm is expanded in the inset. The region is useful to analyze the peptide side because no lipid signal is overlapping $^{2}$. The peaks at 7.35-7.20 and 7.15-6.80 ppm are assigned to the aromatic protons of Phe and Tyr, respectively ${ }^{9,12}$. It is found that the line broadening of the peptide signals is induced by the presence of the vesicle; see the black line. The Phe peaks at $7.25 \mathrm{ppm}$ are shifted to downfield, while the signals around $7.3 \mathrm{ppm}$ slightly move to a high field. These are analogous to the change in the chemical shift when the peptide is added to the micelle of lysophosphatidylcholine ${ }^{9}$. For the Tyr peaks, in contrast, the chemical shifts are almost unaltered even in the presence of the vesicle. The result indicates that L-Enk is oriented with its Phe residue toward to the membrane inside.

The line broadening and the peak shift in Figure 1 imply that L-Enk is bound to the membrane. The bound component of L-Enk is, however, hardly separated from free L-Enk in the 1D NMR spectrum. To separate the bound component from the peptide signal, we apply the PFG technique. The criterion is that the signal assignable to the free peptide preferentially decays with increasing the field gradient (FG) strength to unveil the bound component at the high $\mathrm{FG}^{2}$. In Figure 2 is demonstrated how the signal of L-Enk is attenuated by applying the magnetic field gradient. It is evident that all signals decay in intensity with increasing the FG strength (from top to bottom). Instead, the broad components are uncovered. The intensities of the broad components are less attenuated up to the FG strength of $0.91 \mathrm{~T} / \mathrm{m}$ (the bottom). From these results, the broad components are considered to be bound L-Enk with its motion slowed down in the viscous membrane environment ${ }^{21,25}$. The situation is quite similar to the $5 \mathrm{FU}$ binding reported previously ${ }^{2}$.

\subsection{Kinetics of Binding, Diffusivity, and Binding Amount of L-Enk}

It is found that the bound component of L-Enk is uncovered by ${ }^{1} \mathrm{H}$ NMR in combination with the PFG technique. Therefore, the diffusivity, the kinetics of binding, and the

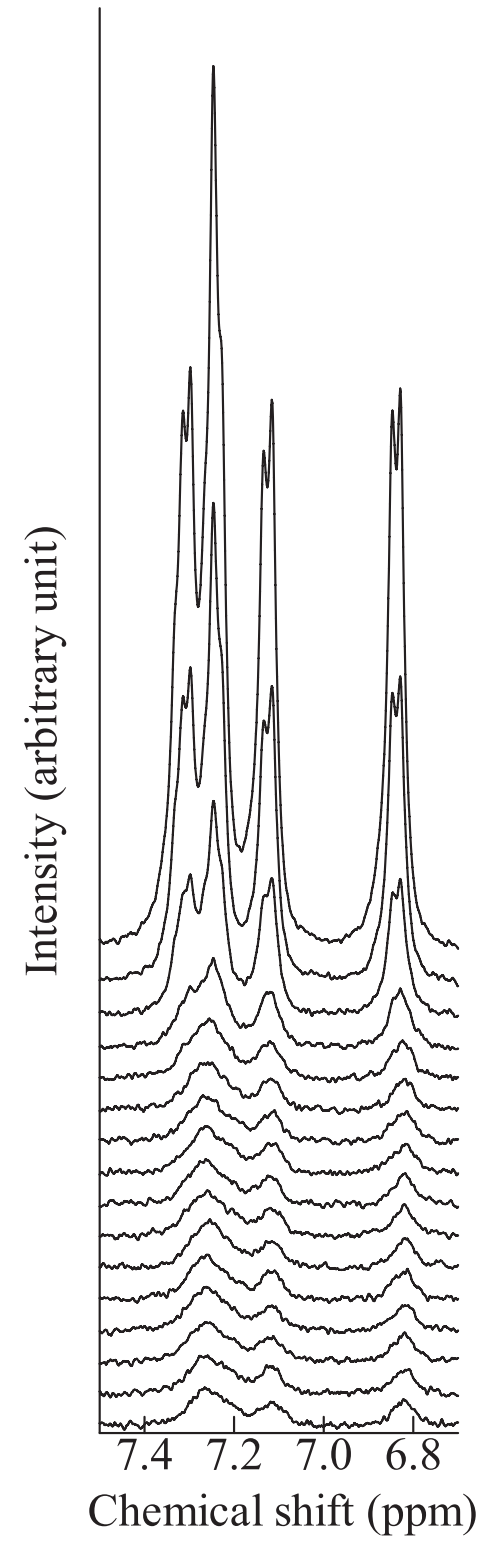

Figure 2 PFG ${ }^{1} \mathrm{H}$ NMR spectra of the aromatic region of L-Enk in the presence of LUV at $303 \mathrm{~K}$. Here, the $16 \mathrm{FG}$ strengths $g=0.05,0.15$, $0.225,0.30,0.35,0.40,0.45,0.50,0.55,0.60,0.65,0.70,0.75,0.80$, $0.85,0.91 \mathrm{~T} / \mathrm{m}$ are applied (from top to bottom) with the pulse width of $0.004 \mathrm{~s}$ at the diffusion time, $0.1 \mathrm{~s}$.

binding amount of L-Enk can be quantified by using the signal intensity decay of the PFG ${ }^{1} \mathrm{H}$ NMR spectrum. We analyze the signal attenuation in accordance with the procedure in section 3.3, by using the peak integral of the aromatic region in Figure 2.

The result is summarized in Figure 3 at various diffusion times, $\Delta_{\text {diff }}$. The circles, triangles, and squares designate the experimental values at $\Delta_{\text {diff }}$ of $0.1,0.5$, and $1.0 \mathrm{~s}$, respectively. The red lines are obtained by fitting Eq. (3) to the experimental values. Each line well reproduces the observed decay of the signal intensities. The dashed black lines are 


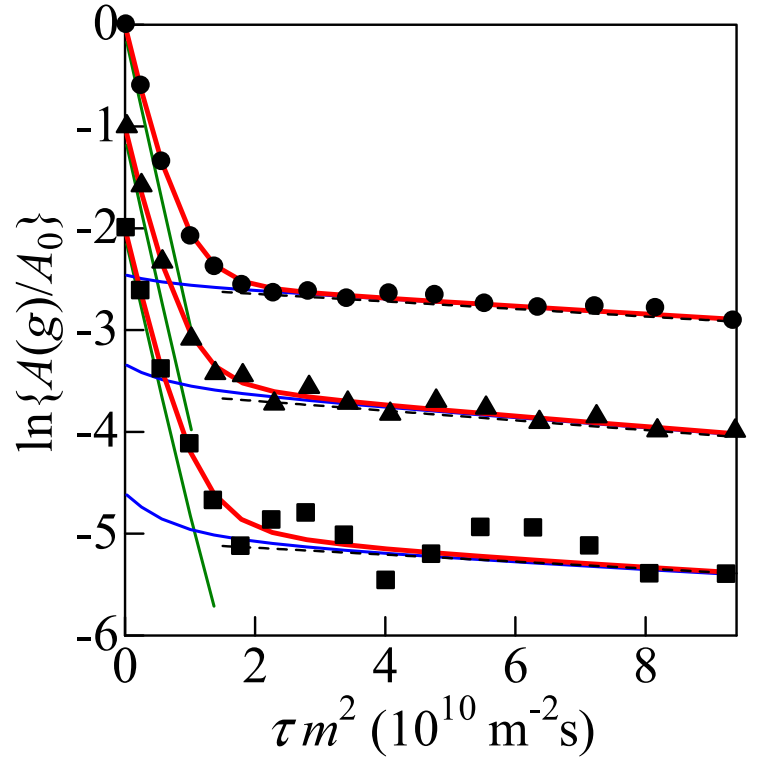

Figure 3 Intensity decay of the L-Enk signal as a function of the FG strength at the diffusion time $\Delta_{\text {diff }}$ of 0.1 (filled circle), 0.5 (filled triangle), and $1.0 \mathrm{~s}$ (filled square). Here the signal intensity is evaluated as the peak integral of the aromatic region in Figure 2. Symbols represent the experimental values. The red lines are obtained by fitting Eq. (3) to the experimental values of peak integrals at the respective $\Delta_{\text {diff }}$. The blue and the green lines demonstrate the decay curves evaluated by Eqs. (10) and (11), respectively. The dashed black lines are the fitting result of the Stejskal-Tanner plot at the large $g$ limit $(g \rightarrow \infty)$, from which the diffusion coefficients of bound L-Enk, $D_{\mathrm{B}}$ are calculated. The results of $\Delta_{\text {diff }}=0.5$ and $1.0 \mathrm{~s}$ are shifted to negative value by -1.0 and -2.0 , respectively.

the fitting results of Eq. (13) to the signal intensity at the large $g$ limit $(g \rightarrow \infty)$. Thus we can evaluate the diffusivity and the rate of the binding and dissociation of L-Enk from Eqs. (3) and (13). It is found that the signal intensity decay is a biexponential function with respect to the FG strength, in contrast to the single exponential decay of L-Enk in water (not shown). The intensities rapidly attenuate at $\mathrm{\tau m}^{2}<$ $2 \times 10^{10} \mathrm{~m}^{-2} \mathrm{~s}$, while slowly at $\mathrm{\tau m}^{2}>2 \times 10^{10} \mathrm{~m}^{-2} \mathrm{~s}$. The nonlinear behavior of the signal attenuation is due to the exchange of the peptide molecule between free and bound states that is similar to the $5 \mathrm{FU}$ binding to the membrane ${ }^{3}$.

The PFG signal of the bound component not only comes from L-Enk bound throughout the time $\Delta_{\text {diff }}$ but also from that transferring from the free to bound states within the time $\Delta_{\text {diff }}$. The transferring peptides are considered to have a large displacement and a large effective diffusion coefficient, because they have undergone a free state during the time $\Delta_{\text {diff }}$. The influence of the transferring peptides leads to the rapid attenuation at $\tau m^{2}<2 \times 10^{10} \mathrm{~m}^{-2} \mathrm{~s}$. On the other hand, the peptide keeping on binding throughout the time $\Delta_{\text {diff }}$ has a small diffusion coefficient, so that the decay of the signal intensity is small. Slow attenuation at $\mathrm{\tau m}^{2}>$ $2 \times 10^{10} \mathrm{~m}^{-2} \mathrm{~s}$ in Figure 3 is due to the contribution of these binding molecules. The decay of the signal intensities has thus two different gradients and becomes non-linear.

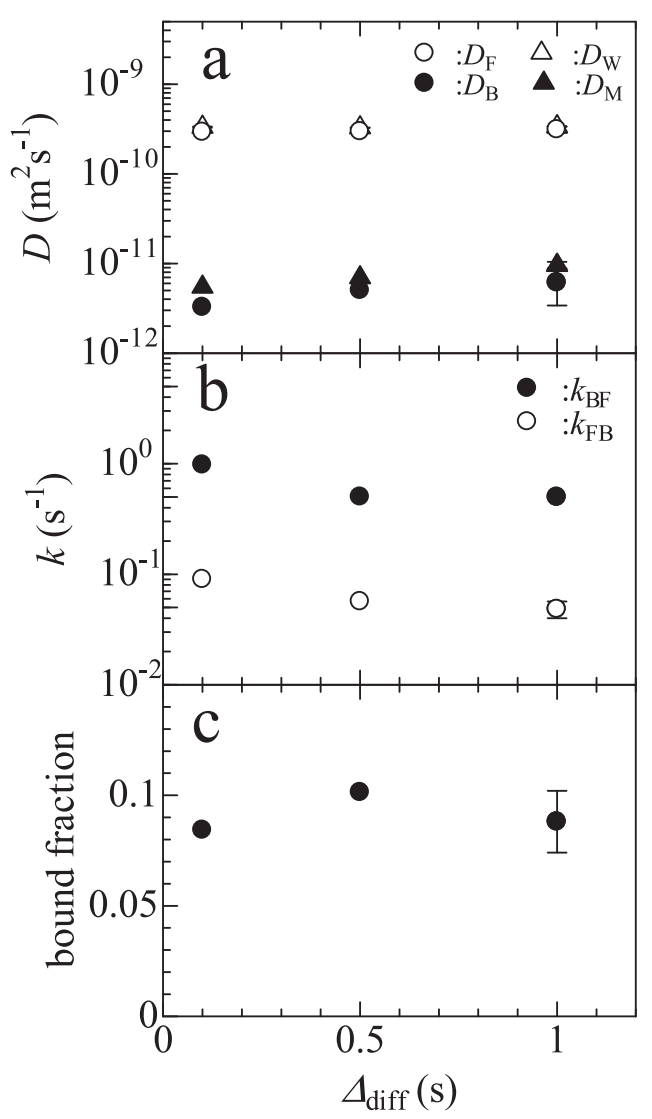

Figure 4 The $\Delta_{\text {diff }}$ dependence of (a) the diffusion coefficients, (b) the rate constants of the binding and dissociation, and (c) the binding amount of L-Enk at $303 \mathrm{~K}$.

The diffusivity of the free and the bound peptide is evaluated by fitting Eqs. (3) and (13) to the experimental $A(g)$ in Figure 3. The fitting of Eq. (3) to the experimental value in the whole range of $g$ gives the diffusion coefficient of the free peptide, $D_{\mathrm{F}}$, together with the rate constants $k_{\mathrm{FB}}$ and $k_{\mathrm{BF}}$. Next, we calculate the diffusion coefficient of the bound peptide, $D_{\mathrm{B}}$, by using the linear relation of the decay plot (Stejskal-Tanner plot) at $g \rightarrow \infty^{3}$; see the dashed black lines. The results are summarized in Figure $4 \mathrm{a}$ as a function of the time $\Delta_{\text {diff }}$. It is found that $D_{\mathrm{F}}$ and $D_{\mathrm{B}}$ give the values of $(2.9 \pm 0.1) \times 10^{-10}$ and $(5.0 \pm 1.4) \times 10^{-12} \mathrm{~m}^{2} / \mathrm{s}$, respectively, at $303 \mathrm{~K}^{26}$. The diffusivity of bound L-Enk is almost 60 times as restricted as free L-Enk. Both values are independent of the time, $\Delta_{\text {diff }}$, indicating the accuracy of the experiment. $D_{\mathrm{F}}$ is in good agreement with the diffusion coefficient of L-Enk in water $\left(D_{\mathrm{W}}=(3.3 \pm 0.1) \times 10^{-10} \mathrm{~m}^{2} / \mathrm{s}\right)$, and $D_{\mathrm{B}}$ corresponds to that of the lipid membrane $\left(D_{\mathrm{M}}=(7.3 \pm 0.7) \times 10^{-12} \mathrm{~m}^{2} / \mathrm{s}\right)$ obtained simultaneously by the EPC signal ${ }^{27}$. All results demonstrate that $D_{\mathrm{F}}$ and $D_{\mathrm{B}}$ are the diffusion coefficients of free and bound L-Enk in the presence of the vesicle.

In Figure $4 \mathrm{~b}$, the rate constants of the binding $\left(k_{\mathrm{FB}}\right)$ and dissociation $\left(k_{\mathrm{BF}}\right)$ of L-Enk are plotted as a function of $\Delta_{\text {diff }}$. The values of $k_{\mathrm{FB}}$ and $k_{\mathrm{BF}}$ are estimated to be $0.040 \pm 0.004$ and $0.40 \pm 0.01 \mathrm{~s}^{-1}$, respectively, and almost independent of 
$\Delta_{\text {diff }}$. The obtained rate constants correspond to the halfvalue periods, $t_{\mathrm{FB}}$ and $t_{\mathrm{BF}}$, of the peptide binding and dissociation,

$$
\tau_{\alpha}=\frac{\ln 2}{k_{\alpha}}, \alpha=\{\mathrm{FB}, \mathrm{BF}\}
$$

as $11.0 \pm 1.0$ and $1.2 \pm 0.1 \mathrm{~s}$. This means that the lifetime of the binding and dissociation of L-Enk is of the order of ten second and a second timescale. Finally, the dissociation constant $K_{\mathrm{d}}$ of the peptide is estimated to be $10.1 \pm 0.8$ from the relation $K_{\mathrm{d}}=k_{\mathrm{BF}} / k_{\mathrm{FB}}$.

To make sure that the obtained rate constants are reasonable, we compare the values of $k_{\mathrm{FB}}$ and $k_{\mathrm{BF}}$ with those of small-sized solutes quantified recently by ${ }^{19} \mathrm{~F} \mathrm{NMR}^{3,4}$. The rate constants of membrane binding and dissociation are 0.2 and $4.1 \mathrm{~s}^{-1}$ for 5FU molecules ${ }^{3}$. For FBPA, $k_{\mathrm{FB}}$ and $k_{\mathrm{BF}}$ are enhanced to $22-280$ and $68-107 \mathrm{~s}^{-1}$ at $303 \mathrm{~K}^{4}$. The time span of the binding and dissociation of $5 \mathrm{FU}$ is $10^{-1}-10^{0} \mathrm{~s}$ timescale $^{3}$, while that of FBPA is decreased to $10^{-3}-10^{-2} \mathrm{~s}^{4}$. This means that the membrane binding of rather hydrophilic $5 F U$ is $2-3$ orders of magnitude slower than that of hydrophobic FBPA. In case of L-Enk, the rate constants $k_{\mathrm{FB}}$ and $k_{\mathrm{BF}}$ are 0.040 and $0.40 \mathrm{~s}^{-1}$. It means that the time span of the binding and dissociation is $10^{0}$ to $10^{1} \mathrm{~s}$ timescale, almost 10 times as slow as 5FU. The larger size and the hydrophilic nature of L-Enk slow down the exchange motion between the bound and free states. It is in marked contrast to the rapid exchange motion of small-sized hydrophobic FBPA on millisecond timescale, the exchange rate of which more than 3 orders of magnitude as large as the peptide.

The bound fraction of L-Enk can also be evaluated by using $K_{\mathrm{d}}$. In Figure $4 \mathrm{c}$ is demonstrated how much of L-Enk is bound to the vesicle. It is found that the bound fraction is $0.09 \pm 0.01$ and almost unaltered throughout the measurement. The result indicates that $0.45 \mathrm{mM}$ of L-Enk is bound to the $40 \mathrm{mM}$ LUV that corresponds to the peptide/lipid molar ratio of 0.011 . Finally, the binding free energy $\Delta G$ of L-Enk from water to LUV is calculated to be $-2.9 \mathrm{~kJ} / \mathrm{mol}$, the magnitude close to the thermal fluctuation, $2.5 \mathrm{~kJ} / \mathrm{mol}$. The value is reasonable in terms of rather hydrophilic nature of L-Enk, that is in sharp contrast to $\Delta G$ of hydrophobic FPBA, $-20 \mathrm{~kJ} / \mathrm{mol}^{4}$.

\section{Conclusions}

In this work, we show how to quantify the dynamics of peptide binding to fluid lipid membranes in situ. The PFG ${ }^{1} \mathrm{H}$ NMR technique is applied for this purpose, using a neuropeptide L-Enk in the presence of LUV with 100-nm diameters. Even in the case that the bound and free states of L-Enk cannot be distinguished in the 1D NMR spectrum, the PFG technique has unveiled the bound component of LEnk after the preferential decay of the free component at the high field gradient. The success is notable because the ${ }^{1} \mathrm{H}$ nucleus is not rare and the method can be a general standard to quantify the dynamics of a variety of peptide bindings. By analyzing the peak integral of the peptide signal as a function of the gradient strength, we have quantified the diffusivity, the kinetics of the binding, and the bound amount of L-Enk by the analytical formula of the Bloch equation with exchange terms.

As compared with the diffusion coefficient of the free LEnk, $2.9 \times 10^{-10} \mathrm{~m}^{2} / \mathrm{s}$, the mobility of the bound L-Enk is slowed down in LUV where the diffusion coefficient of the peptide is $5.0 \times 10^{-12} \mathrm{~m}^{2} / \mathrm{s}$ at $303 \mathrm{~K}$. The rate constants of the binding and dissociation of L-Enk are 0.040 and $0.40 \mathrm{~s}^{-1}$, respectively. This means that the exchange motion of L-Enk between the bound and free states is more than 1-3 orders of magnitude slower than the small-sized 5FU and FBPA. About one tenth of the total L-Enk is bound to the LUV with the peptide/lipid molar ratio of 0.011 . The free energy of the peptide binding is $-2.9 \mathrm{~kJ} / \mathrm{mol}$, the magnitude close to the thermal fluctuation, $2.5 \mathrm{~kJ} / \mathrm{mol}$. This is due to rather hydrophilic nature of L-Enk that is in sharp contrast to the hydrophobic FBPA molecule with the binding free energy of $-20 \mathrm{~kJ} / \mathrm{mol}$.

\section{Acknowledgments}

This work was partly supported by the Grant-in-Aid for Scientific Research (20550027) from JSPS and by the Grantin-Aid for Scientific Research on Innovative Areas (21107527) from MEXT, Japan.

\section{References}

1. Stilbs, P. Fourier Transform Pulsed-gradient spin-echo studies of molecular diffusion. Progress in NMR Spectrosc. 19, 1-45 (1987).

2. Okamura, E. \& Yoshii, N. Drug binding and mobility relating to the thermal fluctuation in fluid lipid membranes. J. Chem. Phys. 129, 215102 (2008).

3. Yoshii, N. \& Okamura, E. Kinetics of membrane binding and dissociation of 5-fluorouracil by pulsed-field-gradient ${ }^{19} \mathrm{~F}$ NMR. Chem. Phys. Lett. 474, 357-361 (2009).

4. Yoshii, N. \& Okamura, E. Binding of hydrophobic fluorinated bisphenol A to large unilamellar vesicles of egg phosphatidylcholine. J. Phys. Chem. B, 115, 11074-11080 (2011).

5. Okamura, E., Ninomiya, K., Futaki,, S., Nagai, Y., Kimura, T., Wakai, C., Matubayasi, N., Sugiura, Y. \& Nakahara, M. Real-time in-cell ${ }^{19} \mathrm{~F}$ NMR study on uptake of fluorescent and nonfluorescent ${ }^{19} \mathrm{~F}$-octaarginines into human Jurkat cells. Chem. Lett. 34, 1064-1065 (2005).

6. Chekmenev, E. Y., Chow, S., Tofan, D., Weitekamp, D. P., Ross, B.D. \& Bhattacharya, P. Fluorine-19 NMR chemical shift probes molecular binding to lipid Membranes. J. Phys. Chem. B 112, 6285-6287 (2008).

7. Stejskal, E. O. \& Tanner, J.E. Spin diffusion measurements: Spin echoes in the presence of a time-dependent field gradient. J. Chem. Phys. 42, 288-292 (1965).

8. Johnson, Jr., C.S. Effects of chemical exchange in diffusionordered 2D NMR spectra. J. Magn. Reson. A 102, 214-218 (1993).

9. Deber, C. M. \& Behnam, B. A. Role of membrane lipids in 
peptide hormone function: Binding of enkephalins to micelles. Proc. Natl. Acad. Sci. USA 81, 61-65 (1984).

10. Sargent, D. F. \& Schwyzer, R. Membrane lipid phase as catalyst for peptide-receptor interactions. Proc. Natl. Acad. Sci. USA 83, 5774-5778 (1986).

11. Jarrell, H.C., Deslauriers, R., McGregor, W.H. \& Smith, I. C. P. Interaction of opioid peptides with model membranes. A carbon-13 nuclear magnetic study of enkephalin binding to phosphatidylserine. Biochemistry 19, 385-390 (1980).

12. Milon, A., Miyazawa, T. \& Higashijima, T. Transferred nuclear Overhauser effect analyses of membrane-bound enkephalin analogues by ${ }^{1} \mathrm{H}$ nuclear magnetic resonance: Correlation between activities and membrane-bound conformations. Biochemistry 29, 65-75 (1990).

13. Graham,W.H., Carter II, E. G. \& Hicks, R. P. Conformational analysis of Met-enkephalin in both aqueous solution and in the presence of sodium dodecyl sulfate micelles using multidimensional NMR and molecular modeling. Biopolymers 32, 1755-1764 (1992).

14. Matsunaga, T. O., Collins, N., Ramaswani, V., Yamamura, S. H., O'Brien, D.F. \& Hruby, V.J. Comparison of the membrane-bound states of two structurally similar $\delta$-selective opioid peptides by transferred nuclear Overhauser effect spectroscopy and molecular modeling. Biochemistry 32, 13180-13189 (1993).

15. D'alagni, M., Delfini, M., Di Nola, A., Eisenberg, M., Paci, M., Roda, L. G. \& Veglia, G. Conformational study of [Met5] enkephalin-Arg-Phe in the presence of phosphatidylserine vesicles. Eur. J. Biochem. 240, 540-549 (1996).

16. Kimura, A., Kuni, N. \& Fujiwara, H. Orientation and conformation of Met-enkephalin in a liquid crystal as studied by magic-angle- and near-magic-angle-spinning two-dimensional NMR spectroscopy. J. Phys. Chem. 100, 14056-14061 (1996).

17. Marcotte, I., Separovic, F., Auger, M. \& Gagné, S. M. A multidimensional ${ }^{1} \mathrm{H}$ NMR investigation of the conformation of methionine-enkephalin in fast-tumbling bicelles. Biophys. $J$. 86, 1587-1600 (2004).

18. Chatterjee, C., Majumder, B. \& Mukhopadhyay, C. Pulsedfield gradient and saturation transfer difference NMR study of enkephalins in the ganglioside GM1 micelle. J. Phys. Chem. $B$ 108, 7430-7436 (2004).
19. Deaton, K. R., Feyen, E. A., Nkulabi, H. J. \& Morris, K.F. Pulsed-field gradient NMR study of sodium dodecyl sulfate micelle-peptide association. Magn. Reson. Chem. 39, 276-282 (2001).

20. Begotka, B. A., Hunsader, J. L., Oparaeche, C., Vincent, J. K. \& Morris, K. F. A pulsed field gradient NMR diffusion investigation of enkephalin peptide-sodium dodecyl sulfate micelle association. Magn. Reson. Chem. 44, 586-593 (2006).

21. Okamura, E., Wakai, C., Matubayasi, N., Sugiura, Y. \& Nakahara, M. Limited slowdown of endocrine-disruptor diffusion in confined fluid lipid membranes. Phys. Rev. Lett. 93, 248101 (2004).

22. Okamura, E., Wakai, C., Matubayasi, N. \& Nakahara, M. ${ }^{14} \mathrm{~N}$ NMR spectra sensitively reflect surface curvature and segmental motion of hydrophilic headgroups in lipid bilayers and micelles. Chem. Lett. 26, 1061-1062 (1997).

23. Wakai, C. \& Nakahara, M. J. Attractive potential effect on the self-diffusion coefficients of a solitary water molecule in organic solvents. Chem. Phys. 106, 7512 (1997).

24. The longitudinal relaxation times were evaluated by the inversion recovery method and the transverse relaxation times were obtained from the half-widths of the signal. Tyr ${ }^{1}$ signals at $6.8 \mathrm{ppm}$ were used.

25. Venable, R.M., Zhang, Y., Hardy, B.J. \& Pastor, R.W. Molecular dynamics simulations of a lipid bilayer and of hexadecane: An investigation of membrane fluidity. Science 262, 223-226 (1993).

26. $D_{\mathrm{B}}$ involves the movement of L-Enk in vesicles and the movement of the vesicle as a whole. When L-Enk is trapped in vesicle for a sufficiently long time, the mean-square displacements of the peptide and the vesicle are coincident with each other in the long-time limit. If the time scale is not long enough, the diffusivity of L-Enk is not necessarily equal to the vesicle diffusion, while it is a certain measure of the molecular mobility in the aggregate.

27. The diffusion coefficient of the lipid is an averaged value determined by using the single exponential decay of the ${ }^{1} \mathrm{H}$ signals of EPC headgroup $\mathrm{N}\left(\mathrm{C}_{3}\right)_{3}$ at $3.21 \mathrm{ppm}$, chain $\mathrm{CH}_{2} \mathrm{CH}=\mathrm{CHCH}_{2}$ at $2.02 \mathrm{ppm}$, and chain $\left(\mathrm{CH}_{2}\right)_{\mathrm{n}}$ at $1.27 \mathrm{ppm}$ with respect to the $\mathrm{FG}$ strength. The signals are designated by the arrows in Figure 1. 